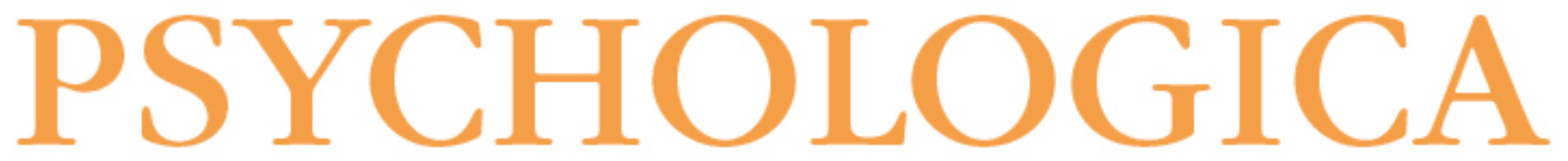

\title{
Representações sociais da saúde e doença mental: um estudo qualitativo com profissionais de saúde mental
}

\author{
Autor(es): $\quad$ Martins, António; Sequeira, Joana \\ Publicado por: Imprensa da Universidade de Coimbra \\ URL \\ persistente: URI:http://hdl.handle.net/10316.2/41361 \\ DOI: $\quad$ DOI:https://doi.org/10.14195/1647-8606_59-2_1 \\ Accessed : $\quad$ 26-Apr-2023 16:30:11
}

A navegação consulta e descarregamento dos títulos inseridos nas Bibliotecas Digitais UC Digitalis, UC Pombalina e UC Impactum, pressupõem a aceitação plena e sem reservas dos Termos e Condições de Uso destas Bibliotecas Digitais, disponíveis em https://digitalis.uc.pt/pt-pt/termos.

Conforme exposto nos referidos Termos e Condições de Uso, o descarregamento de títulos de acesso restrito requer uma licença válida de autorização devendo o utilizador aceder ao(s) documento(s) a partir de um endereço de IP da instituição detentora da supramencionada licença.

Ao utilizador é apenas permitido o descarregamento para uso pessoal, pelo que o emprego do(s) título(s) descarregado(s) para outro fim, designadamente comercial, carece de autorização do respetivo autor ou editor da obra.

Na medida em que todas as obras da UC Digitalis se encontram protegidas pelo Código do Direito de Autor e Direitos Conexos e demais legislação aplicável, toda a cópia, parcial ou total, deste documento, nos casos em que é legalmente admitida, deverá conter ou fazer-se acompanhar por este aviso.

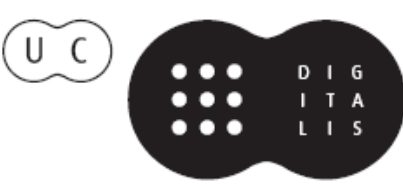


VOLUME

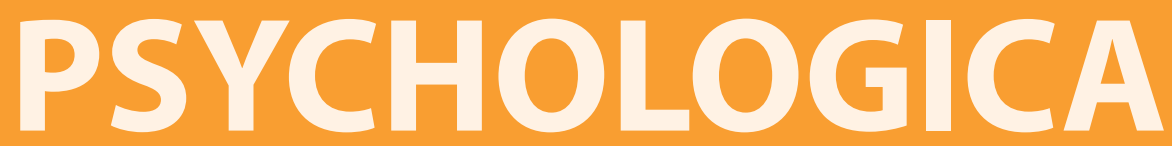

IMPRENSA DA UNIVERSIDADE DE COIMBRA

COIMBRA UNIVERSITY PRESS

FACULDADE DE PSICOLOGIA E DE CIÊNCIAS

DA EDUCAÇÃO DA UNIVERSIDADE DE COIMBRA 


\title{
Representações sociais da saúde e doença mental: Um estudo qualitativo com Profissionais de Saúde Mental
}

\author{
António Martins ${ }^{1}$ e Joana Sequeira ${ }^{2}$
}

Social representations of mental ill-health: A qualitative study with Mental Health Professionals

\begin{abstract}
The aim of this work is to study the social representations of Psychiatrists, Psychiatrist Interns, Child Psychiatrists and Clinical Psychologists about mental health and mental illness. A qualitative and exploratory study was developed based epistemologically on social constructionism and theoretically on social representations. Through the course of the research 30 health professionals participated in a semi-structured interview whose content was analysed by means of the NVivo 10 Programme. The results showed that the social representations of mental illness are heterogeneous, emphasizing operational, etiologic-explanatory and relativist conceptions. Mental health is conceptualized by health professionals according to the flexibility, adaptation, functionality and the biopsychosocial well-being of individuals. Implications of this study to the theoretical framework of social representations of mental health/illness are considered.
\end{abstract}

Keywords: mental illness; mental health; mental health professionals; social representations

1 Instituto Superior Miguel Torga. Email: a_manuel79@hotmail.com

2 Instituto Superior Miguel Torga. Email: joanasequeira@ismt.pt Artigo recebido a 05-08-2015 e aprovado a 15-07-2016. 


\section{Resumo}

Este trabalho pretende conhecer as representações sociais de Psiquiatras, Internos de Psiquiatria, Pedopsiquiatras e Psicólogos Clínicos sobre a saúde e doença mental. É um estudo qualitativo de carácter exploratório, pontuado epistemologicamente pelo construcionismo social e teoricamente pelo quadro das representações sociais. Participaram 30 profissionais de saúde mental, tendo sido realizada uma entrevista semiestruturada que foi analisada quanto ao seu conteúdo - através do programa NVivo 10. Da análise dos resultados, salienta-se que as representações dos profissionais quanto à conceptualização da doença mental são heterogéneas, destacando-se conceções operacionais, etiológico-explicativas e relativistas. A saúde mental é conceptualizada pelos profissionais de saúde em função da flexibilidade, adaptação e funcionalidade e do bem-estar biopsicossocial dos indivíduos. Implicações do presente estudo para o quadro teórico das representações sociais da doença mental são consideradas.

Palavras-chave: doença mental; saúde mental; profissionais de saúde mental; representações sociais

\section{INTRODUÇÃO}

O campo da psicopatologia é caracterizado pela heterogeneidade e incerteza (Morant, 2006), coexistindo múltiplos discursos, teorias e hipóteses sobre os distúrbios psicopatológicos (Pires, 2003). Várias são as teorias e os conceitos elencados para compreender e explicar a doença mental e delinear estratégias terapêuticas (Georgaca, 2001). Modelos biológicos, psicológicos, sociológicos e biopsicossociais concorrem no campo da saúde mental fornecendo grelhas teórico-práticas aos profissionais (Scheid \& Brown, 2010).

O modelo biológico conceptualiza a doença mental como uma doença do cérebro (Cowan, Harter, \& Kandel, 2000; Joffe, 2001). Esta assunção emerge de evidências científicas que apontam as variáveis genéticas da doença mental e dos avanços farmacêuticos das terapias medicamentosas que impulsionaram o conhecimento da química cerebral (Schwartz \& Corcoran, 2010). Também os avanços na neuroanatomia têm permitido correlacionar a estrutura cerebral e o comportamento humano (Schwartz \& Corcoran, 2010).

O modelo psicológico, tradicionalmente, parte do pressuposto de que as perturbações têm uma origem e causalidade psicológica (Gleitman, Fridlund, \& Reisberg, 2011. Segundo Peterson (2010) vários são os modelos psicológicos que oferecem 
um conjunto de asserções sobre a natureza do comportamento humano normal e patológico - por exemplo, o modelo psicanalítico/psicodinâmico, o modelo cognitivo-comportamental e o modelo humanista/existencial.

O modelo sociológico advoga a saúde e doença mental como produto de circunstâncias sociais (Horwitz, 2010; Thoits, 2010), privilegiando os fatores situacionais, comparativamente à omnipresença dos fatores disposicionais presentes nos modelos psicológicos e biológicos (Thoits, 2010). Metaforicamente, os proponentes deste modelo compreendem a doença mental como um colapso do indivíduo perante condições sociais/ambientais adversas (Thoits, 2010).

Por seu turno, o modelo biopsicossocial (Engel, 1977) pressupõe o papel das variáveis biológicas, psicológicas e sociais e a sua articulação como determinantes no processo saúde-doença. Por oposição ao reducionismo psicológico e sociológico, mas principalmente ao reducionismo biológico, surge a proposta biopsicossocial que delimita uma nova base, conceptual e metodológica, para o estudo da saúde e doença (Engel, 1977; Reis, 1999; Ogden, 2004). Propõe uma metateoria subjacente à teoria e prática clínica (Reis, 1999) que considera os mecanismos biológicos, os processos psicológicos e as influências sociais na saúde e doença mental (OMS, 2001; Peterson, 2010).

Os vários modelos descritos exibem múltiplas perspetivas sobre a saúde e doença mental que focam diferentes aspetos, e esse foco determina intervenções e posicionamentos distintos por parte dos profissionais de saúde mental, com implicações na vida dos doentes, famílias e comunidades (Morant, 2006; Walmsley, 2004). Partindo do quadro teórico das representações sociais (Moscovici, 1961), entendidas como "uma modalidade de conhecimento, socialmente elaborada e partilhada, com um objetivo prático e contribuindo para a construção de uma realidade comum a um conjunto social" (Jodelet, 2002, p. 22), verificamos que poucas foram as investigações que tiveram como objeto de estudo as representações sociais de profissionais de saúde mental (Morant 1995, 1998, 2006). Poderemos, no entanto, destacar o trabalho de Zani (1994), que posiciona os Psiquiatras mais próximos do modelo biológico e os Psicólogos mais próximos de conceções psicossociais sobre a saúde e doença mental. Em Nascimento-Schulze, Garcia e Arruda (1995) a saúde é definida pelo sentimento de bem-estar subjetivo, estando associada à ideia de harmonia, e a doença, pelo contrário, conceptualizada como desequilíbrio associado à disfunção do corpo. Com Morant (1995) emergem as representações sociais da doença mental próximas da narrativa biológica e representações sociais funcionais que conceptualizam a doença mental como incapacidade para "funcionar e lidar com".

Assim, é objetivo geral do presente estudo conhecer as representações sociais que os profissionais de saúde mental têm sobre a saúde e doença mental. Especificamente, 
pretende-se: (a) conhecer as principais dimensões de significação, através dos discursos que estruturam as representações dos Psicólogos, Psiquiatras, Pedopsiquiatras e Internos de Psiquiatria sobre o conceito de doença mental e o conceito de saúde mental; (b) verificar as semelhanças e diferenças em função da formação de base dos profissionais.

\section{MÉTODO}

Tipo de estudo

Trata-se de um estudo qualitativo - pois atende à compreensão e aos significados do processo narrativo, de acordo com os atores intervenientes no processo (Coutinho, 2013; Giorgi \& Sousa, 2010) - de carácter exploratório-descritivo - na medida em que pretende a aproximação a uma problemática pouco estudada (Coutinho, 2013).

Assume-se como quadro de referência a teoria das representações sociais (Moscovici, 1961) pontuado epistemologicamente pelo construcionismo social (Gergen, 1985). Sustenta-se, deste modo, que as experiências e os significados não são apenas criados pelo indivíduo, mas existem na cultura e são influenciados por ela (Gergen, 1985; Quartilho, 2001), constituindo a narrativa ou a representação social um elo de ligação entre a experiência individual e o sistema sociocultural.

\section{Participantes}

A seleção dos participantes obedeceu aos critérios da amostragem por conveniência (Coutinho, 2013). Os critérios de inclusão foram: a formação de base dos profissionais de saúde mental a exercer clínica em contexto institucional público ou privado. Participaram 30 profissionais de saúde mental, 13 Psicólogos Clínicos e 10 Médicos Psiquiatras, dois Médicos Pedopsiquiatras e cinco Médicos Internos de Psiquiatria. Treze são do sexo masculino e 17 do sexo feminino. Dos Psicólogos, três participantes são do sexo masculino e 10 são do sexo feminino. Dos Psiquiatras, oito são do sexo masculino e dois do sexo feminino. Dos Pedopsiquiatras, um é do sexo masculino e outro 
feminino. Dos Internos de Psiquiatria, um é do sexo masculino e quatro do sexo feminino.

A idade média desses profissionais é de 39 anos $(D P=10.79)$, sendo que o mais novo tem 25 anos e o mais velho tem 60. No grupo dos Psicólogos a idade média é de $38(D P=10.48)$, dos Psiquiatras $44(D P=11.68)$, dos Pedopsiquiatras $37(D P=0.71)$ e dos Internos de Psiquiatria $29(D P=3.03)$. Quanto à escolaridade, 14 participantes são licenciados, seis mestres e 10 doutores. Quanto aos Psicólogos três têm licenciatura, dois mestrado e oito têm doutoramento. Dos Psiquiatras sete têm licenciatura, um mestrado e dois têm doutoramento. Quanto aos Pedopsiquiatras dois têm licenciatura. Dos Internos de psiquiatria dois têm licenciatura e três têm mestrado.

Tabela 1

Dados Sociodemográficos de uma Amostra de Profissionais de Saúde Mental

\begin{tabular}{|c|c|c|c|c|c|c|c|c|c|c|}
\hline & \multicolumn{2}{|c|}{$\begin{array}{l}\text { Total } \\
\mathrm{N}=30\end{array}$} & \multicolumn{2}{|c|}{$\begin{array}{l}\text { Psicólogos } \\
\mathrm{N}=13\end{array}$} & \multicolumn{2}{|c|}{$\begin{array}{l}\text { Psiquiatras } \\
\mathrm{N}=10\end{array}$} & \multicolumn{2}{|c|}{$\begin{array}{l}\text { Pedopsiq. } \\
\mathrm{N}=2\end{array}$} & \multicolumn{2}{|c|}{$\begin{array}{l}\text { Int. Psiq. } \\
\mathrm{N}=5\end{array}$} \\
\hline & $M$ & $D P$ & $M$ & $D P$ & $M$ & $D P$ & $M$ & $D P$ & $M$ & $D P$ \\
\hline \multirow[t]{2}{*}{ Idade } & 38.53 & 10.79 & 38.31 & 10.48 & 44.10 & 11.68 & 36.50 & 0.71 & 28.8 & 3.03 \\
\hline & $\mathrm{N}$ & $\%$ & $\mathrm{~N}$ & $\%$ & $\mathrm{~N}$ & $\%$ & $\mathrm{~N}$ & $\%$ & $\mathrm{~N}$ & $\%$ \\
\hline \multicolumn{11}{|l|}{ Género } \\
\hline Masculino & 13 & 43.3 & 3 & 23.1 & 8 & 80 & 1 & 50 & 1 & 20 \\
\hline Feminino & 17 & 56.7 & 10 & 76.9 & 2 & 20 & 1 & 50 & 4 & 80 \\
\hline \multicolumn{11}{|l|}{ Escolaridade } \\
\hline Licenciatura & 14 & 46.7 & 3 & 23.1 & 7 & 70 & 2 & 100 & 2 & 40 \\
\hline Mestrado & 6 & 20 & 2 & 15.4 & 1 & 10 & 0 & 0 & 3 & 60 \\
\hline Doutoramento & 10 & 33.3 & 8 & 61.5 & 2 & 20 & 0 & 0 & 0 & 0 \\
\hline
\end{tabular}

Nota: $\mathrm{N}$ = número total de sujeitos; $\mathrm{M}$ = Média; $\mathrm{DP}$ = Desvio de Padrão; Int. Psiq. = Internos de Psiquiatria.

\section{Instrumentos}

Tendo em consideração a especificidade dos objetivos, o enquadramento epistemológico e o referencial teórico, foram construídos dois instrumentos para recolha de dados: o questionário sociodemográfico - que permitiu a caracterização dos participantes quanto às variáveis sociodemográficas, profissionais e académicas - idade dos participantes, sexo, atividade profissional, graduação académica e local onde exerce a atividade profissional - e a entrevista semiestruturada - que se traduziu em duas questões de carácter aberto: (1) O que entende por doença mental? (2) $\mathrm{O}$ que entende por saúde mental? 


\section{Procedimentos}

A recolha de dados realizou-se entre Janeiro e Junho de 2013. Num primeiro período o questionário sociodemográfico e profissional e a entrevista semiestruturada foram construídos e testados com dois especialistas da área da psicologia e psiquiatria. O segundo período correspondeu à aplicação do questionário e da entrevista aos participantes da investigação. Nos dois períodos da investigação, a entrevista semiestruturada foi gravada em suporte digital, à exceção de um participante que respondeu por escrito às questões da entrevista. Posteriormente, as entrevistas foram transcritas para o processador de texto Word. Nenhuma expressão dos entrevistados/participantes foi modificada.

Foi solicitada, aos diretores de serviço de psiquiatria, permissão para recolha de dados no espaço institucional - Centro Hospitalar e Universitário de Coimbra e Centro Hospitalar Baixo Vouga.

Os participantes foram contactados via e-mail, solicitando a colaboração no estudo, tendo depois sido agendado um encontro com os mesmos no seu local de trabalho. A recolha de dados contou sempre com a presença do investigador e os participantes deram o seu consentimento informado e voluntário ficando assegurado o anonimato dos participantes e a confidencialidade dos dados.

O tempo de duração de aplicação do protocolo variou entre os 15 minutos e os 50 minutos.

\section{Análise de dados}

A técnica utilizada no processo de análise de dados foi a análise de conteúdo (Bardin, 2011; Vala, 2009), compreendendo as orientações de Vala (2009) - (1) delimitação dos objetivos e definição de um quadro teórico de referência, (2) constituição do corpus, (3) definição de categorias e (4) definição de unidades de análise. Depois da construção da entrevista semiestruturada, recolha dos dados e respetiva transcrição, procedeu-se, numa primeira fase, à leitura flutuante. De seguida foi efetuada uma leitura pormenorizada dos dados e identificadas as categorias temáticas. Todo este processo contou com o apoio do programa de análise de dados qualitativos NVIVO10.

As entrevistas semiestruturadas foram codificadas separada e individualmente por dois juízes que acordaram quanto às categorias emergentes. De referir, ainda, que para a análise e tratamento dos dados recolhidos através do questionário sociodemográfico e profissional, e com o intuito de se proceder à análise estatística descritiva da amostra, foi utilizado o programa informático de análise estatística Statistical Package for the Social Sciences (SPSS), versão 19.0. 


\section{RESULTADOS}

\section{Conceções sobre a doença mental}

Atendendo à conceptualização de "Doença Mental” (Tabela 2), a categoria emergente mais referenciada é "Perturbação", que conta com 36 referências de 20 entrevistados. Aqui a doença mental é contextualizada de forma equivalente a "alteração", "transtorno", "limitação" ou "incapacidade" que condiciona e limita o funcionamento (seja ele "psicológico", "neurobiológico", "biopsicossocial" ou "adaptativo"). "A doença mental é pela sua própria condição alguma coisa que perturba, que está localizado na mente e que perturba o funcionamento adequado do sujeito, segundo ele próprio, ou segundo o feedback que recebe da interação social, da família, etc.” (E13, Psicólogo).

O "mal-estar e sofrimento", com oito referências de sete participantes, é a segunda categoria mais saliente. "Tem a ver com sofrimento, mal-estar, com uma busca de qualquer coisa.” (E11, Psicólogo).

A categoria "Desvio da normalidade estatística" surge referenciada por quatro vezes por quatro participantes.

Doença mental para mim é um desvio da normalidade comportamental, cognitiva e emocional, que se traduz, de facto, por uma diferença também estatística da normalidade, mas também no sentido biológico, ou seja as doenças mentais terão subjacentes anomalias, de facto, do Sistema Nervoso Central (E3, Psiquiatra).

Pontuando a atividade subjetiva e intersubjetiva, psicológica e social da doença mental, emerge a categoria "Relatividade Psicológica e Sociocultural", com sete referências totais por cinco participantes. Sendo que, das sete referências, três referências correspondem a dois Psiquiatras e outras três a dois Psicólogos, verificando-se uma distribuição homogénea entre os dois grupos profissionais. "O que é patologia ou doença mental num determinado sítio pode não o ser num outro local dependendo do enquadramento que lhe é dado." (E9, Psicólogo).

Outro conceito que emerge na estrutura das conceções de doença mental é o de doença mental como "ausência de saúde", com duas referências de dois participantes, um Psiquiatra e um Psicólogo. "A saúde mental (...) um bem-estar psicológico, social, etc. a todos os níveis, sem dúvida quando existe doença mental há esta ausência de bem-estar também a vários níveis.” (E21, Psicólogo). 
Em resumo, observamos que o campo conceptual da doença mental é múltiplo, complexo e heterogéneo. No entanto, no grupo dos psicólogos, destacam-se conceções operacionais, neutras do ponto de vista teórico, com pontos de ancoragem à definição de doença mental do DSM-IV-R (APA, 2006). No grupo dos Psiquiatras e dos Internos de Psiquiatria destacam-se conceções etiológico-explicativas, comprometidas do ponto de vista teórico com o modelo biomédico. No grupo dos psiquiatras, sobressaem ainda conceções relativistas sobre a doença mental.

Tabela 2

Conceções sobre a Doença Mental

\begin{tabular}{|c|c|c|c|c|c|c|c|c|c|c|}
\hline \multirow{3}{*}{ Doença Mental } & \multicolumn{10}{|c|}{ Dimensão Conceptual } \\
\hline & \multicolumn{4}{|c|}{ Entrevistados } & \multirow[t]{2}{*}{ Total } & \multicolumn{4}{|c|}{ Referências } & \multirow[t]{2}{*}{ Total } \\
\hline & Psiq & Psic & Int & Pedo & & Psiq & Psic & Int & Pedo & \\
\hline \multicolumn{11}{|l|}{ Perturbação } \\
\hline Psicológica & 3 & 6 & 1 & 0 & 10 & 3 & 6 & 2 & 0 & 11 \\
\hline Neurobiológica & 2 & 2 & 1 & 0 & 5 & 3 & 8 & 2 & 0 & 13 \\
\hline Adaptativa & 1 & 2 & 0 & 1 & 4 & 1 & 4 & 0 & 2 & 7 \\
\hline Biopsicossocial & 0 & 0 & 1 & 0 & 1 & 1 & 0 & 0 & 0 & 1 \\
\hline Total Parcial & & & & & & & & & & 32 \\
\hline Mal-estar e sofrimento & 2 & 4 & 1 & 0 & 7 & 2 & 5 & 1 & 0 & 8 \\
\hline Relatividade Psic. e SocioCult. & 2 & 2 & 1 & 0 & 5 & 3 & 3 & 1 & 0 & 7 \\
\hline Desvio da normalidade estatística & 3 & 0 & 1 & 0 & 4 & 3 & 0 & 1 & 0 & 4 \\
\hline Ausência de Saúde Mental & 1 & 1 & 0 & 0 & 2 & 1 & 0 & 1 & 0 & 2 \\
\hline Total & & & & & & & & & & 55 \\
\hline
\end{tabular}

Nota : Psiq = Psiquiatra Psic $=$ Psicólogo Int = Interno de Psiquiatria Pedo = Pedopsiquiatra; Relatividade Psic. e Socio-Cult. = Relatividade Psicológica e Sociocultural.

\section{Conceções sobre a saúde mental}

No que diz respeito à saúde mental (Tabela 3), a maioria dos participantes enquadra-a no sentido mais amplo da saúde, ou seja, como um "bem-estar" integral ou biopsicossocial, que se traduz na subcategoria "Bem-estar biopsicossocial", que conta com 20 referências de 16 participantes. Destas 20 referências, 10 foram feitas por oito Psiquiatras, seis por quatro Psicólogos, três por três Internos de Psiquiatria e um por um Pedopsiquiatra.

Entendo por saúde mental uma saúde integrada, também não existe só saúde mental, então nesse aspeto faria mais sentido falar em saúde do contexto da definição da OMS, de um bem-estar integrado que é social, que é psicológico, que é 
físico e portanto a saúde mental é uma parte desse todo, mas ninguém tem saúde mental se não tiver saúde nos vários outros campos, nas várias outras áreas da vida (E11, Psicólogo).

Filiada à categoria "Bem-estar", para além da referida subcategoria "Bem-estar Biopsicossocial", surgem duas outras subcategorias: o "Bem-estar Psicossocial" e o "Bem-estar Psicológico".

O "Bem-estar Psicossocial" apresenta sete referências de seis participantes emerge da constatação do processo de funcionamento harmonioso do indivíduo no seu ambiente social, promotor de um encaixe adaptativo indivíduo-meio. Das sete referências, cinco são de quatro Internos de Psiquiatria, uma de um Psiquiatra e uma de um Pedopsiquiatra.

Então a saúde mental seria de uma forma assim mais resumida, o conjunto de condições que o indivíduo tem do ponto de vista psicológico que o permite fazer a sua vida de uma forma adaptada, a ele, aos outros e à sociedade (E12, Psiquiatra).

O "Bem-estar Psicológico" conta com um total de cinco referências de cinco participantes - duas referências de dois Internos de Psiquiatria, duas de dois Psicólogos e uma de um Pedopsiquiatra. "Saúde mental acho que tem mais a ver com o bem-estar psicológico. O facto de se sentir bem.” (E15, Psicólogo).

Associada à estrutura conceptual "Bem-estar" emerge a categoria "Flexibilidade, adaptação e funcionalidade" com 21 referências por 11 participantes, salientando-se o grupo profissional dos Psicólogos com 20 referências de nove participantes. "[O indivíduo] é tão mais saudável quanto mais capaz for de construir multi-respostas tão alternativas quanto possível, numa multi-realidade quanto possível num processo sistemático." (E7, Psicólogo).

Da saúde mental perspetivada negativamente, emerge a conceção de saúde mental como "Ausência de Doença", com nove referências de oito participantes. Quatro emergem do discurso de quatro Psicólogos, três de três Psiquiatras e duas de um Interno de Psiquiatria.

Em oposição ao que é a minha perspetiva da doença mental, portanto, a saúde mental será o oposto, ou seja, neste caso, num sentido estrito, a ausência dessas anomalias de facto biológicas do SNC que correspondem à ou melhor, às doenças mentais (E3, Psiquiatra).

Do ter ao não ter saúde mental, da qualidade ou falta dela, surge a Saúde Mental como um "continuum saúde-doença", com quatro referências de quatro Psicólogos, 
duas de dois Internos de Psiquiatria e um de um Psiquiatra, o que contabiliza sete referências de sete participantes. "A diferença entre saúde e doença não é qualitativa, mas é mais quantitativa, isto é, as fronteiras muitas vezes são ténues, são esbatidas e portanto é mais uma questão do quão saudável está ou quão doente está." (E30, Interno de Psiquiatria).

Se para alguns participantes a doença mental é um conceito complexo, para outros a saúde mental é ainda mais difícil de conceptualizar. Neste sentido, emerge a categoria "Conceito Lato e Complexo" com seis referências de cinco participantes. Destas, três referem-se a três Psiquiatras e as outras três a dois Internos de Psiquiatria. "É mais difícil definir saúde do que doença." (E29, Psiquiatra). A questão da saúde "é muito mais ampla, muito mais lata que a doença." (E30, Interno de Psiquiatria).

Para um Psiquiatra, que conta com três referências, a questão da saúde desloca-se do domínio da ciência num sentido estrito para o conceito definido politicamente. "Saúde mental é um conceito, enfim, falado muito pela organização mundial de saúde e por políticas de saúde mais do que por instituições de saúde (...) envolve mais questões não médicas, questões também muito políticas.” (E14, Psiquiatra).

Sintetizando, constatamos que a saúde mental é fundamentalmente conceptualizada pelos profissionais de saúde em função do equilíbrio psicossocial do indivíduo - função da flexibilidade, adaptação e funcionalidade - e do bem-estar biopsicossocial.

Tabela 3

Conceções sobre Saúde Mental

\begin{tabular}{|c|c|c|c|c|c|c|c|c|c|c|}
\hline \multirow{3}{*}{ Saúde Mental } & \multicolumn{10}{|c|}{ Dimensão Conceptual } \\
\hline & \multicolumn{4}{|c|}{ Entrevistados } & \multirow[t]{2}{*}{ Total } & \multicolumn{4}{|c|}{$\underline{\text { Referências }}$} & \multirow[t]{2}{*}{ Total } \\
\hline & Psiq & Psic & Int & Pedo & & Psiq & Psic & Int & Pedo & \\
\hline \multicolumn{11}{|l|}{ Bem-estar } \\
\hline Biopsicossocial & 8 & 4 & 3 & 1 & 16 & 10 & 6 & 3 & 1 & 20 \\
\hline Psicossocial & 0 & 2 & 2 & 1 & 5 & 1 & 0 & 5 & 1 & 7 \\
\hline Psicológico & 1 & 0 & 4 & 1 & 6 & 0 & 2 & 2 & 1 & 5 \\
\hline Total Parcial & & & & & & & & & & 32 \\
\hline Flexibilidade, adaptação e func. & 1 & 9 & 1 & 1 & 12 & 1 & 20 & 1 & 1 & 23 \\
\hline Ausência de doença & 3 & 4 & 1 & 0 & 8 & 3 & 4 & 2 & 0 & 9 \\
\hline Continuum saúde-doença & 1 & 4 & 2 & 0 & 7 & 1 & 4 & 2 & 0 & 7 \\
\hline Conceito Lato e complexo & 3 & 0 & 2 & 0 & 5 & 3 & 0 & 3 & 0 & 6 \\
\hline Conceito Político & 1 & 0 & 0 & 0 & 1 & 3 & 0 & 0 & 0 & 3 \\
\hline Total & & & & & & & & & & 80 \\
\hline
\end{tabular}

Notas: Psiq = Psiquiatra Psic = Psicólogo Int = Interno de Psiquiatria $;$ Pedo = Pedopsiquiatra; Flexibilidade, adaptação e func. $=$ Flexibilidade, adaptação e funcionalidade 


\section{DISCUSSÃO}

Considerando a doença mental e as representações sociais dos profissionais de saúde mental, salientamos o seu carácter heterogéneo que, tal como nos estudos de Morant $(1995,2006)$, expõem a incerteza e ambiguidade do conceito. Estas representações são, pelo menos, teoricamente expectáveis, sobretudo, devido ao facto de o universo conceptual da doença mental ser construído sob múltiplas visões sobre o que significa doença mental (Morant, 2006; Rothes, 2006). Trata-se de um universo não consensual, onde predominam, pelo cariz complexo do objeto de referência, representações polémicas que conduzem ao debate e negociação constantes entre os profissionais de saúde mental (Cabecinhas, 2009; Morant, 2006; Vala, 2006). Neste sentido, encontramos frequentemente nas narrativas dos participantes, representações da doença mental como perturbação, alteração, transtorno, limitação ou incapacidade.

Não obstante a heterogeneidade, algumas temáticas comuns emergiram dos dados. De salientar:

(1) a doença mental como perturbação psicológica e geradora de mal-estar e sofrimento, que reflete uma conceção de cariz operacional, não etiológica, com pontos de ancoragem à definição de doença mental do DSM-IV-TR (2006), que a conceptualiza como

uma síndrome ou padrão comportamentais ou psicológicos clinicamente significativos que ocorrem num sujeito e que estão associados com ansiedade actual (por exemplo, um sintoma doloroso) ou incapacidade (por exemplo, incapacidade em uma ou mais áreas importantes do funcionamento). Qualquer que seja a sua causa original, deve ser considerada como uma manifestação de uma disfunção comportamental, psicológica ou biológica no sujeito.” (p. xxxi).

Esta definição tem um valor fundamental para os participantes deste estudo na medida em que operacionaliza o conceito e permite, através de processos sociocognitivos, transformar um fenómeno comportamental não familiar e complexo em familiar e inteligível (Flick, Fischer, Schwarz, \& Walter, 2002; Foster, 2001). Embora esta conceção seja proeminente na maioria dos profissionais consultados, não é contudo consensual, nem tão pouco na literatura científica (Wakefield, 1992, 2007), onde o debate ontológico e epistemológico se prolonga no tempo sobre o que é a doença mental, o que não é ou se é mesmo algo ou alguma coisa. Deste modo, a validade conceptual do conceito proposto pelos autores do DSM-IV-TR (2006) e a própria doença mental é questionada (Aragona, 2009; Wakefield, 1992, 2007); 
(2) a doença mental com substrato neurobiológico emerge maioritariamente das narrativas dos médicos. Trata-se de uma conceção etiológico-explicativa. Aqui, a doença mental é sinónimo de doença do e no cérebro. Esta tese, mais consensual na biomedicina, privilegia explicações organicistas (Nordenfelt, 2007; Quartilho, 2001). A doença mental como uma alteração do funcionamento neurobiológico, encontrada neste estudo, tem correspondência no estudo de Zani (1994) e de Morant (1995), que sugerem que os psiquiatras estão mais próximos do modelo biológico;

(3) a doença mental dependente e relativa aos contextos socioculturais. No sentido inverso, alguns psiquiatras participantes consideram que a doença mental é conceptualizada em função dos contextos sociais e dos processos de significação que aí ocorrem. Esta linha de raciocínio é extensamente debatida na comunidade científica e filosófica (Aragona, 2009; Moncrieff, 2010; Szasz, 1978; Wakefield, 1992, 2006, 2007), na medida em que se questiona se o conceito de doença mental é um conceito científico, objetivo e universal ou um conceito sociopolítico relativo à dinâmica espaço-tempo. Este debate surge frequentemente polarizado: de um dos lados, os defensores da doença como facto (científico) e, do outro, os que percebem a doença como dependente dos valores (sociais).

No entanto, algumas tentativas de elaboração de um conceito híbrido emergiram entre diversos autores, de onde destacamos Wakefield (1992, 2006, 2007), que conceptualiza a doença mental como uma disfunção prejudicial - disfunção, refere-se à incapacidade de o sistema psicofisiológico desempenhar a sua função. Prejudicial refere-se à desadequação num contexto social particular (Aragona, 2009; Wakefield, 1992, 2006, 2007). Vemos, deste modo, que a incerteza conceptual que existe no campo da doença mental tem também o seu paralelo nas narrativas dos participantes.

Sobre as conceções acerca da Saúde Mental salienta-se duas representações entre os participantes. A saúde como bem-estar biopsicossocial, mais comum nos Psiquiatras e a saúde como flexibilidade, adaptação e funcionalidade, mais comum nos Psicólogos.

A saúde como bem-estar biopsicossocial vai ao encontro dos resultados de outros estudos qualitativos sobre as representações sociais da saúde e da doença, nomeadamente os de Nascimento-Schulze et al. (1995) com médicos e Flick et al. (2002) com médicos e enfermeiros. Neste quadro de referência, a saúde é biológica, psicológica e social no seu todo (Peterson, 2010). Não faz sentido, para grande parte destes profissionais, falar de saúde simplesmente como ausência de doença, nem tão pouco de saúde biológica, ou de saúde psicológica, ou de saúde social, por si só. A saúde é um processo biopsicossocial. Este quadro enunciativo sugere como ponto de ancoragem o conceito de saúde da Organização Mundial de Saúde (OMS). Esta organização define saúde como o estado de bem-estar físico, mental e social, total, e não apenas a ausência de doença (OMS, 2001) e surge, de alguma forma, como contraponto à conceção negativa de saúde (Almeida Filho \& Jucá, 2002), 
como ausência de doença (Dalgalarrondo, 2008) ou como silêncio do corpo ou dos órgãos (Loureiro, 2008). No entanto, a conceção de saúde como completo bem-estar biopsicossocial e não simplesmente como ausência de doença (OMS, 2001), por um lado, é um conceito muito criticável, por ser lato, complexo, impreciso e, consequentemente, difícil de definir e objetivar (Dalgalarrondo, 2008). Por outro lado, permanece, segundo Nordenfelt (2007), idealista e utópico, na medida em que poucas pessoas se encaixariam na categoria saudável.

A saúde como flexibilidade, adaptação e funcionamento encontra o seu referente na visão de saúde como equilíbrio do indivíduo perante si, na sua relação com os outros e com o ambiente, refletindo a ideia de harmonia (Nordenfelt, 2007). Esta narrativa é encontrada nos resultados globais do estudo, já referido, de Nascimento-Schulze et al. (1995), onde as narrativas emergentes se enquadram exatamente na noção referida de equilíbrio e harmonia.

\section{CONCLUSÕES}

Os estudos das representações sociais sobre a saúde e doença mental têm normalmente como foco as racionalidades leigas (Foster, 2001; Jodelet, 1989; Loureiro, 2008). $\mathrm{O}$ presente estudo permitiu ir além das racionalidades leigas e conhecer as narrativas sociais dos conceitos de saúde e doença mental de especialistas da área da saúde mental. Dos dados recolhidos e analisados pudemos constatar dimensões distintas sobre a doença mental: operacionais, não etiológicas, enfatizando o mal-estar e sofrimento; e etiológicas-explicativas, que salientam o determinismo psicológico, neurobiológico ou social na génese da doença mental. As narrativas sobre a saúde mental também são heterogéneas, com pontos de ancoragem ao modelo biopsicossocial da saúde e a características como flexibilidade e equilíbrio psicossocial. Estes dados são importantes, pois permitem observar de que forma os modelos teóricos moldam as representações dos profissionais de saúde mental. Esta influência dos modelos teóricos tem o poder de tornar inteligível o processo de saúde-doença, balizando-o narrativamente do ponto de vista cognitivo e social. Estes resultados poderão constituir-se como contributo para a investigação na área das representações sociais, na medida em que se expõe as diferenças conceptuais entre os profissionais da área, o que apela à reflexão e questionamento da teoria e prática clínica. Por fim, tendo em conta a importância do estudo das representações sociais, seria importante, no futuro, estudar, por um lado, a congruência entre as representações sociais dos profissionais de saúde mental e a prática clínica efetiva e, por outro lado, estudar a forma como as representações sociais dos profissionais moldam as representações sociais leigas. 


\section{REFERÊNCIAS}

Almeida-Filho, N., \& Jucá, V. (2002). Saúde como ausência de doença: Crítica da teoria funcionalista de Christopher Boorse. Ciência e Saúde Coletiva, 7(4), 879-889. doi: 10.1590/s1413-81232002000400019

Aragona, M. (2009). The concept of mental disorder and the DSM-V. Dialogues in Philosophy, Mental and Neuro Sciences, 2(1), 1-14.

Associação Americana de Psiquiatria (APA). (2006). Manual de diagnóstico e estatística das perturbações mentais ( $4^{\mathrm{a}}$ ed.). Lisboa: Climepsi Editores.

Bardin, L. (2011). Análise de conteúdo (4ª ed.). Lisboa: Edições 70.

Cabecinhas, R. (2009). Investigar representações sociais: Metodologias e níveis de análise. In M. M. Baptista (Eds.), Cultura: Metodologias e investigação (pp. 51-66). Lisboa: Verso Edições.

Coutinho, C. P. (2013). Metodologia de investigação em ciências sociais e humanas: Teoria e prática ( $2^{a}$ ed.). Coimbra: Almedina.

Cowan, W. M., Harter, D. H., \& Kandel, E. R. (2000). The emergence of modern neuroscience: Some implications for neurology and psychiatry. Annual Review of Neuroscience, 23, 343-391. doi:10.1146/annurev.neuro.23.1.343

Dalgalarrondo, P. (2008). Psicopatologia e semiologia dos transtornos mentais (2a ed.). São Paulo: Artmed Editora.

Engel, G. L. (1977). The need for a new medical model: A challenge for biomedicine. Science, 196(4286), 129-136. doi: $10.1126 /$ science. 847460

Flick, U., Fischer, C., Schwartz, F. W., \& Walter, U. (2002). Social representations of health held by health professionals: The case of general practitioners and home-care nurses. Social Science Information, 41(4), 581-602. doi: 10.1177/0539018402041004005

Foster, J. (2001). Unification and differentiation: A study of the social representations of mental illness. Papers on Social Representations, 10, 1-18. Consultado em http://www.psych.lse.ac.uk/ psr/PSR2001/10_3Foste.pdf

Georgaca, E. (2001). O discurso da psicopatologia: Uma abordagem crítica ao dispositivo teórico da psiquiatria. In M. M. Gonçalves \& O. F. Gonçalves (Eds.), Psicoterapia, narrativa e discurso: A construção conversacional da mudança (pp. 331-373). Coimbra: Quarteto.

Gergen, K. J. (1985). The social constructionist movement in modern psychology. American Psychologist, 40(3), 266-275. doi: 10.1037/0003-066X.40.3.266

Giorgi, A., \& Sousa, D. (2010). Método fenomenológico de investigação em psicologia. Lisboa: Fim de Século.

Gleitman, H., Fridlund, A., \& Reisberg, D. (2011). Psicologia (9a ed.). Lisboa: Fundação Calouste Gulbenkian.

Horwitz, A. V. (2010). An overview of sociological perspectives on the definitions, causes, and responses to mental health and illness. In T. L. Scheid \& T. N. Brown (Eds.), A handbook for the study of mental health ( $2^{\text {nd }}$ ed., pp. 6-19). New York: Cambridge University Press.

Jodelet, D. (1989). Folies et représentations sociales. Paris: PUF.

Jodelet, D. (2002). Representações sociais: Um domínio em expansão. In D. Jodelet (Eds.), As representações sociais (pp. 17-44). Rio de Janeiro: Eduerj.

Joffe, R. T. (2001). Progress in the biology of psychiatry. Journal of Psychiatry and Neuroscience, 26(2), 101-102. Consultado em https://www.ncbi.nlm.nih.gov/pmc/articles/PMC1407759/pdf/ jpn00090-0015.pdf

Loureiro, L. (2008). Representações sociais da loucura: Importância para a promoção da saúde mental (Tese de doutoramento não publicada). Universidade do Porto, Porto. 
Moncrief, J. (2010). Psychiatric diagnosis as a political device. Social Theory and Health, 8(4), 370-382. doi: $10.1057 /$ sth.2009.11

Morant, N. (1995). What is mental illness? Social representations of mental illness among British and French mental health professionals. Papers on social representations, 4(1), 41-52. Consultado em http://www.psych.lse.ac.uk/psr/PSR1995/4_1995Moran.pdf

Morant, N. (1998). The social representation of mental ill-health in communities of mental health practitioners in the UK and France. Social Science Information, 37(4), 663-685. doi: 10.1177/053901898037004006

Morant, N. (2006). Social representations and professional knowledge: The representation of mental illness among mental health practitioners. British Journal of Social Psychology, 45(4), 817-838. doi: 10.1348/014466605X81036

Moscovici, S. (1961) La psychanalyse, son image et son publique. Paris: PUF.

Nascimento-Schulze, C. M., Garcia, A. I. F., \& Arruda, D. C. (1995). Health paradigms, social representations of health and illness and theirs central nucleus. Papers on Social Representations, 4(2), 187-198. Consultado em http://www.psych.lse.ac.uk/psr/PSR1995/4_1995Nasci.pdf

Nordenfelt, L. (2007). The concepts of health and illness revisited. Medicine, Health Care, and Philosophy, 10(1), 5-10. doi: 10.1007/s11019-006-9017-3

Ogden, J. (2004). Psicologia da Saúde (2a ed.). Lisboa: Climepsi Editores.

OMS (Organização Mundial de Saúde). (2001). Saúde mental, nova concepção, nova esperança: Relatório sobre a Saúde no Mundo. Consultado a 30 Julho $2013 \mathrm{em} \mathrm{http://www.who.int/whr/2001/en/}$ whr01_djmessage_po.pdf

Peterson, C. (2010). Psychological approaches to mental illness. In T. L. Scheid \& T. N. Brown (Eds.), A handbook for the study of mental health ( $2^{\text {nd }}$ ed., pp. 89-105). New York: Cambridge University Press.

Pires, C. M. L. (2003). Manual de psicopatologia (2a ed.). Leiria: Editorial Diferença.

Quartilho, M. (2001). Cultura, medicina e psiquiatria: Do sintoma à experiência. Coimbra: Quarteto Editora.

Reis, J. (1999). Modelo metateórico da psicologia da saúde para o séc. XXI: Interacção ou integração biopsicossocial? Análise Psicológica, 3(XVII), 415-433. Consultado em http://www.scielo.mec. $\mathrm{pt} / \mathrm{pdf} / \mathrm{aps} / \mathrm{v} 17 \mathrm{n} 3 / \mathrm{v} 17 \mathrm{n} 3 \mathrm{a} 02 . \mathrm{pdf}$

Rothes, I. (2006). Suicídio juvenil: Representações sociais dos médicos e dos psicólogos (Dissertação de mestrado não publicada). Universidade do Porto, Porto.

Scheid, T. L., \& Brown, T. N. (Eds.). (2010). Handbook for the study of mental health ( $2^{\text {nd }}$ ed.). New York: Cambridge University Press.

Schwartz, S., \& Corcoran, C. (2010). Biological theories of psychiatric disorders: A sociological approach. In T. L. Scheid \& T. N. Brown (Eds.), A handbook for the study of mental health ( $2^{\text {nd }}$ ed., pp. 64-88). New York: Cambridge University Press. doi.org/10.1017/CBO9780511984945

Silva, A. (2003). Formação, percursos e identidades. Coimbra: Quarteto Editora.

Szasz, T. (1978). A fabricação da loucura: Um estudo entre a inquisição e o movimento de saúde mental ( $2^{a}$ ed.). Rio de Janeiro: Zahar.

Thoits, P. A. (2010). Sociological approaches to mental illness. In T. L. Scheid \& T. N. Brown (Eds.), A handbook for the study of mental health (2 ${ }^{\text {nd }}$ ed., pp. 106-124). New York: Cambridge University Press.

Vala, J (2006). Representações sociais e psicologia social do conhecimento quotidiano. In J. Vala \& M. B. Monteiro (Eds.), Psicologia social (7a ed., pp. 457-502). Lisboa: Fundação Calouste Gulbenkian.

Vala, J. (2009). A análise de conteúdo. In A. S. Silva \& J. M. Pinto (Eds.), Metodologia das ciências sociais (15ª ed., pp. 101-128). Porto: Edições Afrontamento. 
Wakefield, J. C. (1992). The concept of mental disorder: On the boundary between biological and social values. American Psychologist, 47(3), 373-388. doi: 10.1037/0003-066X.47.3.373

Wakefield, J. C. (2006). What makes a mental disorder mental? Philosophy, Psychiatry, e Psychology, 13(2), 123-131. doi: 10.1353/ppp.2007.0010

Wakefield, J. C. (2007). The concept of mental disorder: Diagnostic implications of the harmful dysfunction analysis. World Psychiatry, 6(3), 149-156. Consultado em https://www.ncbi.nlm. nih.gov/pmc/articles/PMC2174594/

Walmsley, C. J. (2004). Social representation and the study of professional practice. International Journal of Qualitative Methods, 3(4), 78-103. Consultado em https://journals.library.ualberta. ca/ijqm/index.php/IJQM/article/view/4380/3566

Zani, B. (1994). Social representations of mental illness: Lay and professional perspectives. In G. M. Breakwell \& D. V. Canter (Eds.), Empirical approaches to social representations (pp. 315-330). Oxford: Clarendon Press. 